Acta Crystallographica Section D

\section{Biological Crystallography}

ISSN 0907-4449

\section{Jiawei Wang, ${ }^{a}$ Alexander Wlodawer ${ }^{\mathrm{b}}$ and Zbigniew Dauter $^{\mathrm{C} *}$}

asAIC-Frederick Inc., Basic Research Program, Argonne National Laboratory, Argonne, IL 60439, USA, '⿳亠丷厂 Protein Structure Section, MCL, National Cancer Institute, NCl-Frederick, Frederick, MD 21702, USA, and 'Synchrotron Radiation Research Section, MCL, National Cancer Institute, Argonne National Laboratory, Argonne, IL 60439, USA

Correspondence e-mail: dauter@anl.gov

\title{
What happens when the signs of anomalous differences or the handedness of substructure are inverted?
}

Proper solution of a macromolecular crystal structure based on anomalous scattering and/or isomorphous differences requires that the anomalous differences in reflection amplitudes be measured properly and that the correct enantiomer of the substructure be selected. If this information is wrong then the resulting electron-density maps will not show the correct structural features, but the reflection phases and map features will be related to the correct ones in a specific way. This text aims to explain how misinterpretation of the Bijvoet differences or of the substructure affects the resulting phases and electron-density maps.

\section{Introduction}

Recently, Matthews (2007) published an excellent and thorough discussion of the principles of phasing macromolecular crystal structures based on the anomalous diffraction signal, showing that change of the signs of anomalous differences or misinterpretation of the handedness of the constellation of anomalous scatterers will never lead to correctly interpretable electron-density maps. His article is a reaction to the recent retraction of erroneous structures resulting from the wrong interpretation of experimental diffraction data, leading to inversion of the signs of anomalous differences (Chang et al., 2006; Miller, 2007).

As Matthews correctly stated, phasing methods based on the anomalous diffraction signal will only lead to the correct structure if the reflections are indexed properly (in the righthanded coordinate system) and if the handedness of the constellation of anomalous scatterers, one of two possibilities, is correctly chosen. A special case occurs if the substructure has a centrosymmetric arrangement, where the problem of its handedness vanishes. However, the phases and electrondensity maps obtained with wrongly assigned anomalous differences or handedness of the substructure are related to the correct solution in a specific way. These relationships will be elaborated with emphasis on the single-wavelength anomalous diffraction (SAD) method of phasing.

\section{SAD phasing}

\subsection{Correct interpretation}

Fig. 1 illustrates the relationships between various contributions to a structure factor if the crystal contains atoms displaying an anomalous scattering effect (for simplicity, it is assumed that all anomalous scatterers are of the same kind). The contribution of the normally scattering part is represented by the brown vector $F_{\mathrm{N}}$ and the normal scattering of the anomalous atoms by the red vector $F_{\mathrm{A}}$. The real part of the anomalous scattering is represented by the vector $F_{\mathrm{A}}^{\prime}$ and the
Received 1 May 2007

Accepted 25 May 2007
(C) 2007 International Union of Crystallography Printed in Denmark - all rights reserved 
imaginary part by the purple vectors $F_{\mathrm{A}}^{\prime \prime}$. They result from the anomalous corrections to the atomic form factor: $f_{\mathrm{A}}=f_{\mathrm{A}}^{\mathrm{o}}+f_{\mathrm{A}}^{\prime}+$ $i f_{\mathrm{A}}^{\prime \prime}$. The vector $F_{\mathrm{A}}^{\prime}$ is collinear and antiparallel to $F_{\mathrm{A}}$, since $f_{\mathrm{A}}^{\prime}$ is real and usually negative. The purple vector $+F_{\mathrm{A}}^{\prime \prime}$ is perpendicular and rotated anticlockwise with respect to $F_{\mathrm{A}}$, since the correction $f_{\mathrm{A}}^{\prime \prime}$ is multiplied by the imaginary factor $i=(-1)^{1 / 2}$. The purple vector $-F_{\mathrm{A}}^{\prime \prime}$ represents the anomalous contribution of the negative Friedel mate or, more strictly, it represents the complex conjugate of the Friedel-related reflection, which in fact has an opposite phase and lies on the other side of the horizontal axis of the Argand diagram. If it were drawn as the original reflection, not its mirror image, the vector $F_{\mathrm{A}}^{\prime \prime}(-h)$ would also be rotated anticlockwise with respect to $F_{\mathrm{A}}(-h)$. The black vectors $F_{\mathrm{T}}^{+}$and $F_{\mathrm{T}}^{-}$therefore represent the total structure factor of a pair of Friedel-related reflections. Only the lengths of these two vectors (reflection amplitudes) are available in a single-wavelength diffraction data set. The anomalous difference $\Delta F^{ \pm}$is equal to the difference between the two amplitudes within a Friedel (or Bijvoet) pair. A more detailed discussion of the relevant vector and phase relations can be found elsewhere (Blundell \& Johnson, 1976; Drenth, 1999; Dauter, 2002).

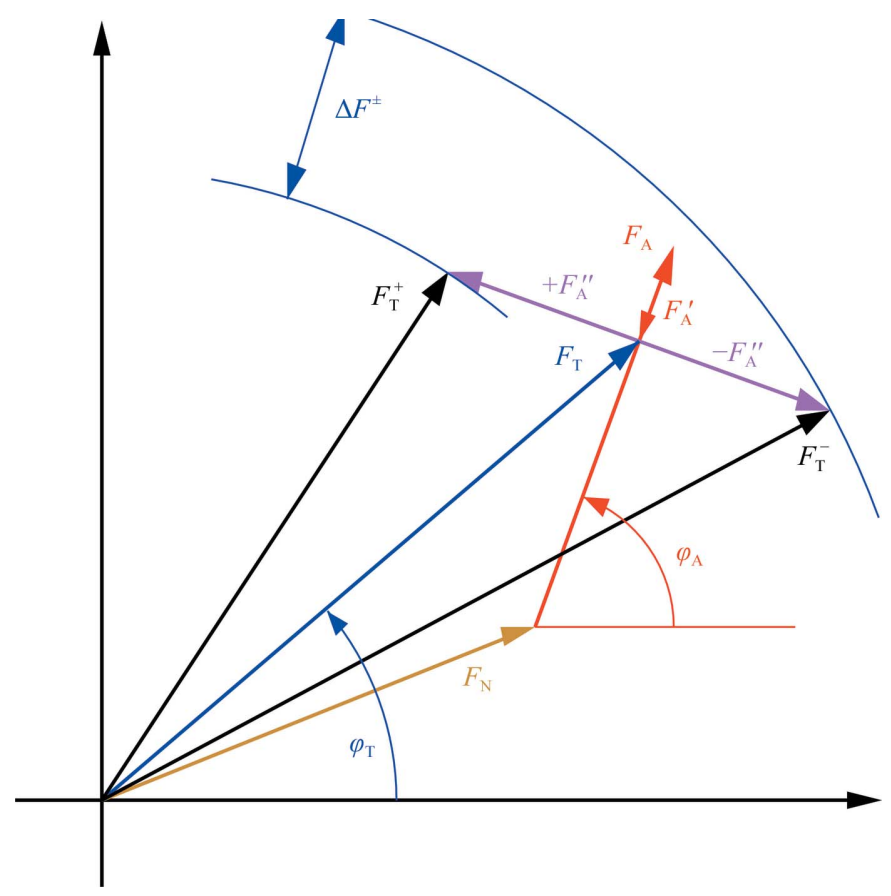

Figure 1

An Argand diagram illustrating the structure factor of a reflection with significant anomalous scattering contribution. The brown vector represents the contribution of normally scattering atoms, $F_{\mathrm{N}}$; the red and purple vectors relate the normal, $F_{\mathrm{A}}, F_{\mathrm{A}}^{\prime}$, and anomalous, $\pm F_{\mathrm{A}}^{\prime \prime}$, contributions of the anomalously scattering substructure for two Friedel mates; the two black vectors show the total structure factors for each of the Friedel mates, $F_{\mathrm{T}}^{+}$and $F_{\mathrm{T}}^{-}$; the blue vector shows the total average structure factor $F_{\mathrm{T}}$. In the SAD experiment only the amplitudes (lengths) of $F_{\mathrm{T}}^{+}$and $F_{\mathrm{T}}^{-}$are available (the ends of black vectors must lie on the circles of appropriate radii), but if the anomalous substructure is known, the size and orientation of the red/purple vectors can be calculated. The purple $\pm F_{\mathrm{A}}^{\prime \prime}$ vectors must end up on the corresponding circles. To calculate the electron-density map, the total structure factor should be used, with amplitude $\left|F_{\mathrm{T}}\right| \simeq\left(\left|F_{\mathrm{T}}^{+}\right|+\left|F_{\mathrm{T}}^{-}\right|\right) / 2$ and phase $\varphi_{\mathrm{T}}$.
The measurable anomalous difference $\Delta F^{ \pm}$is approximately related to the total anomalous scattering contribution $2 F_{\mathrm{A}}^{\prime \prime}$ through the sinusoidal function, depending on the difference in the directions of the total average structure factor $F_{\mathrm{T}}$ and of the partial structure factor representing the anomalous substructure (blue and red vectors in Fig. 1): $\Delta F^{ \pm}=\left|F^{+}\right|-\left|F^{-}\right| \simeq 2 F_{\mathrm{A}}^{\prime \prime} \sin \left(\varphi_{\mathrm{T}}-\varphi_{\mathrm{A}}\right)=2 \delta_{\mathrm{A}} F_{\mathrm{A}} \sin \left(\varphi_{\mathrm{T}}-\varphi_{\mathrm{A}}\right)$, where $\delta_{\mathrm{A}}=F_{\mathrm{A}}^{\prime \prime} / F_{\mathrm{A}}$ is characteristic for a particular scatterer. In principle, to locate the positions of anomalous scatterers one should use the amplitudes $\left|F_{\mathrm{A}}\right|$ representing the total contribution of the anomalous substructure. These amplitudes may be estimated in the multi-wavelength anomalous diffraction (MAD) experiment, although not from a single SAD data set. However, because the measurable anomalous differences are related to the $F_{\mathrm{A}}$ values, it is possible to use them for the location of anomalous sites using Patterson or direct methods. Since $\sin ^{2}(\alpha)=[1-\cos (2 \alpha)] / 2$, the Patterson synthesis calculated with $\left|\Delta F^{ \pm}\right|^{2} \simeq 4 \delta_{\mathrm{A}}^{2} F_{\mathrm{A}}^{2} \sin ^{2}\left(\varphi_{\mathrm{T}}-\varphi_{\mathrm{A}}\right)=2 \delta_{\mathrm{A}}^{2} F_{\mathrm{A}}^{2}-$ $2 \delta_{\mathrm{A}}^{2} F_{\mathrm{A}}^{2} \cos \left[2\left(\varphi_{\mathrm{T}}-\varphi_{\mathrm{A}}\right)\right]$ will reproduce the proper vectors representing the anomalous scatterers (first term) accompanied by (more-or-less random) noise resulting from the second cosine term. Location of the anomalous scatterer sites is the first step in the SAD phasing protocol.

If the anomalous substructure is located, it is possible to calculate its contribution; therefore, the length and orientation of the red and purple vectors are known. In addition to this, the lengths, but not the directions, of the total amplitudes of both Friedel mates are available. It is therefore required that the ends of the (correctly oriented) purple vectors $+F_{\mathrm{A}}^{\prime \prime}$ and $-F_{\mathrm{A}}^{\prime \prime}$ must lie on the two circles with radii corresponding to $\left|F_{\mathrm{T}}^{+}\right|$and $\left|F_{\mathrm{T}}^{-}\right|$. As illustrated in Fig. 2(a), since $\sin \left(\varphi_{\mathrm{T}}-\varphi_{\mathrm{A}}\right)=$ $\sin \left(\pi-\varphi_{\mathrm{T}}+\varphi_{\mathrm{A}}\right)$, there are two solutions to this geometrical problem, giving rise to the well known phase ambiguity inherent to SAD (as well as SIR) phasing (Drenth, 1999).

The two solutions in the SAD approach are not absolutely symmetrical, as is evidenced by the different lengths of the brown vectors $F_{\mathrm{N}}$ corresponding to the normal scatterers. In effect, the anomalous and normal substructures represent different fractions of the total crystal in the two possible phase solutions, which increases the probability that the solution with smaller $F_{\mathrm{N}}$ and a smaller angle between $F_{\mathrm{A}}$ and $F_{\mathrm{T}}$ is correct (Ramachandran \& Raman, 1956). If the anomalous substructure is small, this influence of the known (anomalous) partial structure, described by $\operatorname{Sim}(1959,1964)$, is very small. In the simplest approach, one can accept the average phase $\varphi_{\mathrm{SAD}}=\varphi_{\mathrm{A}}-\pi / 2$ for calculation of the initial electron-density map. As pointed out by Wang (1985), such a map will correspond to a superposition of the correct image of the structure (contributed by the set of correctly chosen phases) and of the featureless noisy map (resulting from the contribution of the wrong phases). This map, although not necessarily directly interpretable, may at least indicate which parts of the cell correspond to the macromolecule and which to the much flatter solvent regions. The subsequent iterative densitymodification procedures (such as solvent flattening) should enhance the correct features and indicate the correct choice of the alternative SAD phase. In conclusion, the properly 
indexed data set and correctly chosen handedness of the substructure lead to the SAD map showing the positively contoured features of the macromolecule of correct chirality, with $\mathrm{L}$-amino acids in proteins or D-sugars in nucleic acids.

\subsection{Substructure of opposite handedness}

In the SAD approach the anomalous scatterer sites can be located either by analysis of the Patterson syntheses or by the use of direct methods, with both methods utilizing anomalous differences. Neither of these methods differentiates between the two possible enantiomorphic constellations of atoms in the substructure. Both outcomes are equally probable and both have to be checked for correctness. If the Patterson or directmethods solution correctly represents the anomalous substructure in the crystal, the initial SAD map should disclose correct (although not very accurate) features of the crystal structure. However, the opposite handedness of the substructure results in a different set of phases and a map with different features.

This phenomenon is illustrated in Fig. 2(b). All vectors representing various scattering contributors have the same length as previously, but the phase $\varphi_{\mathrm{A}}$ of the anomalous substructure is negated, corresponding to the opposite enantiomer. As a consequence, the two alternative phase solutions are different and the resulting vector $F_{\mathrm{SAD}}^{\mathrm{c}}$ lies on the other side of the vertical axis of the Argand diagram, with the corresponding phase $\varphi_{\mathrm{SAD}}^{\mathrm{c}}=\pi-\varphi_{\mathrm{SAD}}^{\mathrm{o}}$. Since $\cos (\pi-\varphi)=$ $-\cos (\varphi)$, this has the following consequence for the calculated Fourier map:

$$
\begin{aligned}
\rho^{\mathrm{c}}(r) & =\sum^{N}\left|F_{h}\right| \exp \left(i \varphi_{h}^{\mathrm{c}}\right) \exp (-2 \pi i h \cdot r) \\
& =2 \sum^{N / 2}\left|F_{h}\right| \cos \left(-2 \pi h \cdot r+\varphi_{h}^{\mathrm{c}}\right) \\
& =2 \sum^{N / 2}\left|F_{h}\right| \cos \left[2 \pi h \cdot(-r)+\pi-\varphi_{h}^{\mathrm{o}}\right] \\
& =-2 \sum^{N / 2}\left|F_{h}\right| \cos \left[-2 \pi h \cdot(-r)+\varphi_{h}^{\mathrm{o}}\right] \\
& =-\rho^{\mathrm{o}}(-r) .
\end{aligned}
$$

The Fourier synthesis will therefore be related to the correct map by centrosymmetric inversion and will have negative values. It will show the structural features of inverted chirality in the negative contours of the map.

Since the positive contours of such a map do not show any significant features except noise and the solvent-flattening procedure removes all negative features, such a map will not lead to solution of the crystal structure.

\subsection{Data with inverted signs of anomalous differences}

If the indices of Friedel-related reflections are inadvertently exchanged before SAD phasing, the resulting SAD phase $\varphi_{\mathrm{SAD}}^{\mathrm{i}}$ will be increased by $\pi$ in comparison with the original,

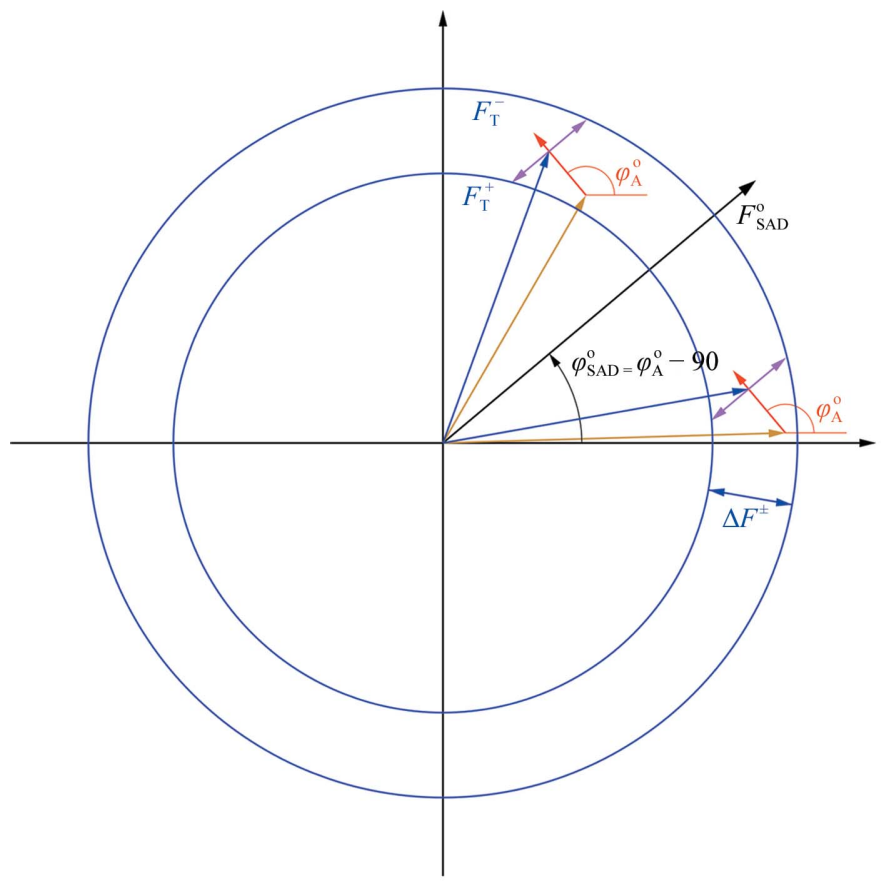

(a)

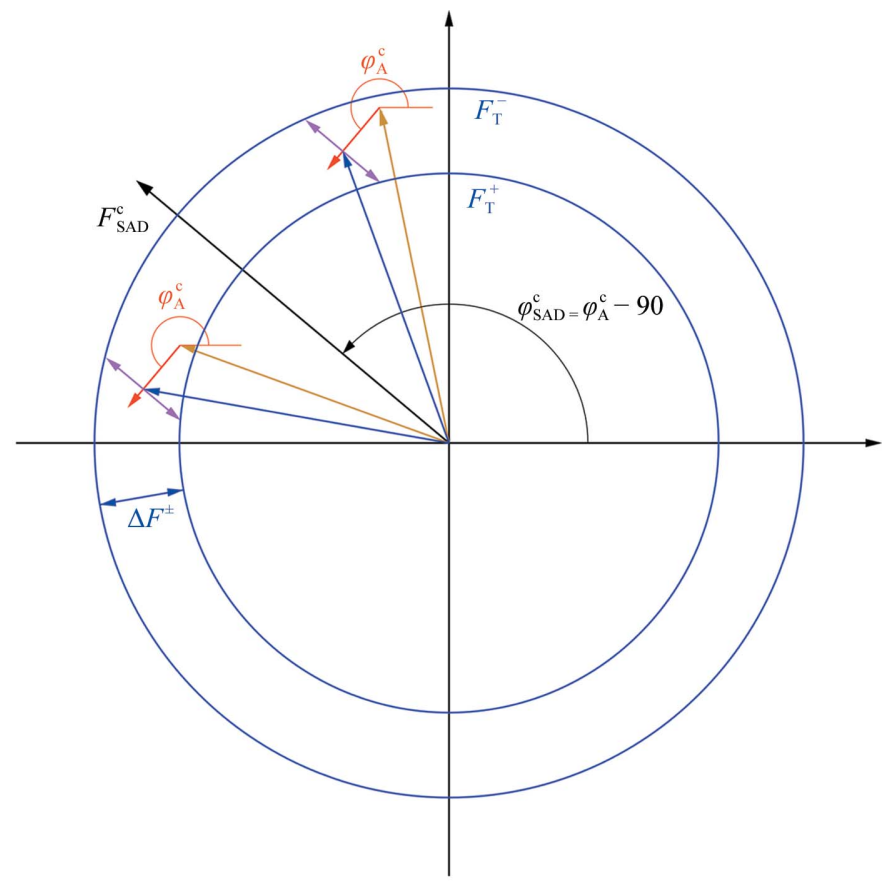

(b)

Figure 2

An illustration of SAD phasing. All vectors are colored as in Fig. 1. (a) If only single-wavelength data are available and the anomalous substructure is located, there are two possible solutions for the structure-factor phase. Since it is not known a priori which alternative is correct, the average phase $\varphi_{\mathrm{SAD}}^{\mathrm{O}}$ is used with the average amplitude (weighted by the figure of merit, which corresponds to the cosine of the half-angle between the two possible total blue vectors). The average (black) vector $F_{\mathrm{SAD}}$ is always parallel to the purple vectors representing the anomalous scattering contribution of the substructure. (b) If the handedness of the substructure is inverted through the center of symmetry, the phase of the substructure is reversed, $\varphi_{\mathrm{A}}^{\mathrm{c}}=-\varphi_{\mathrm{A}}^{\mathrm{o}}$, which results in different alternative solutions of the SAD phases; in effect, the average SAD phase changes, $\varphi_{\mathrm{SAD}}^{\mathrm{c}}=\pi-\varphi_{\mathrm{SAD}}^{\mathrm{o}}$. 
correct phase $\varphi_{\mathrm{SAD}}^{\mathrm{o}}$, as illustrated in Fig. 2(c). Since $\cos (\pi+\varphi)$ $=-\cos (\varphi)$, the map calculated with such phases will have the following relation to the original map:

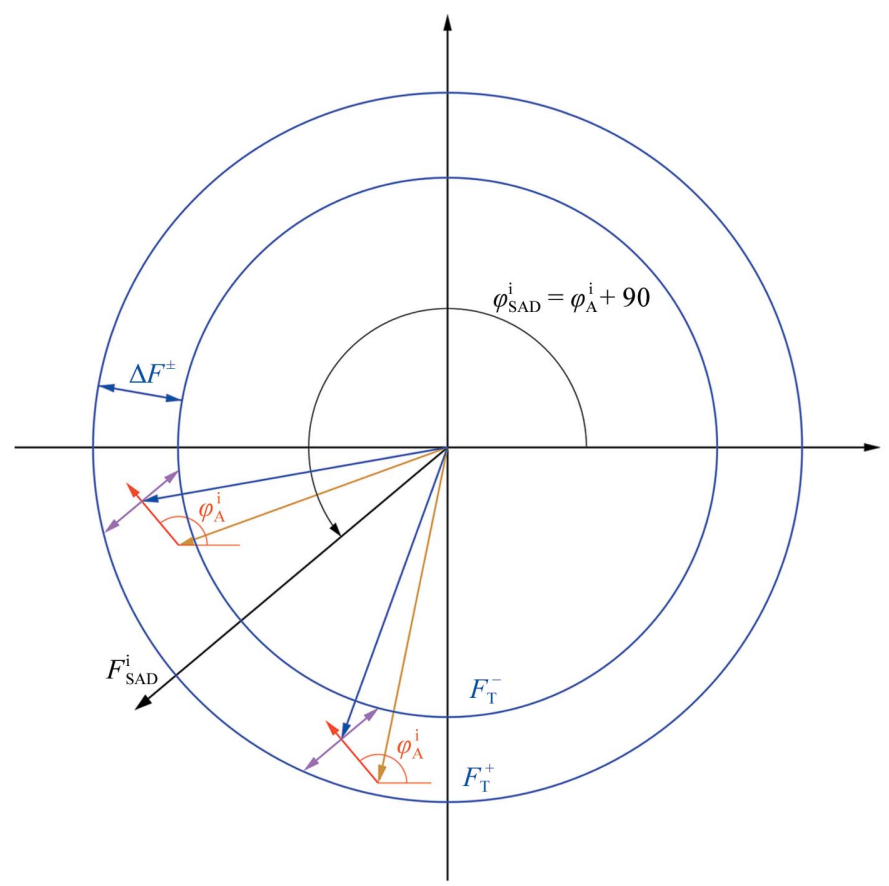

(c)

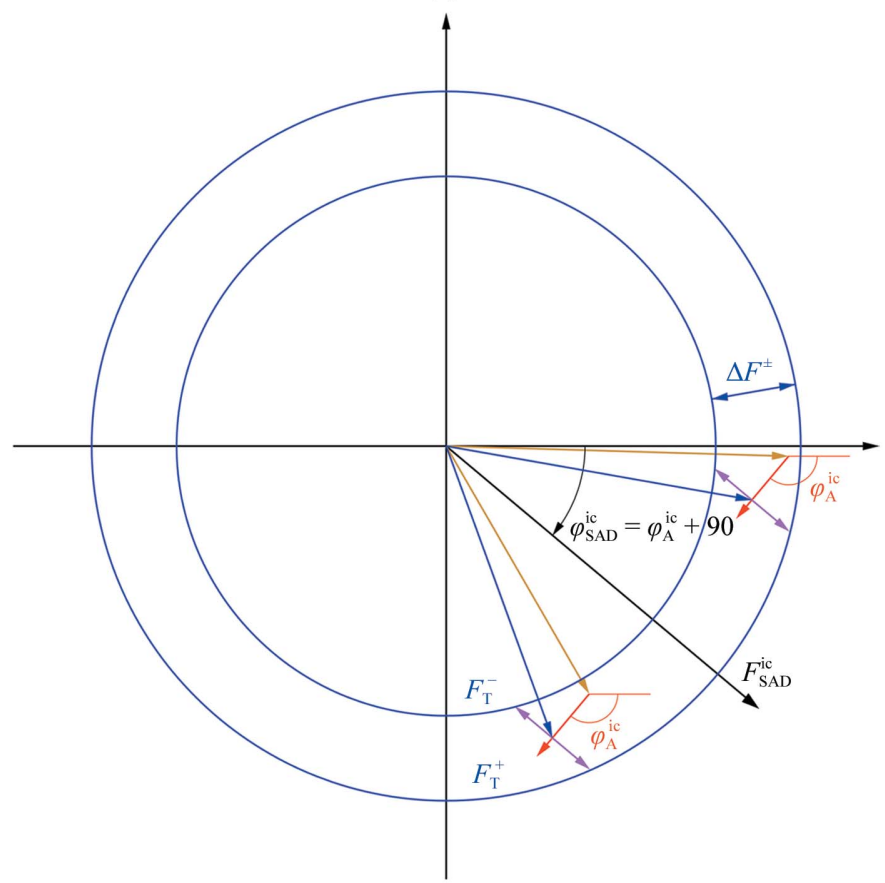

$(d)$

$$
\begin{aligned}
\rho^{\mathrm{i}}(r) & =\sum^{N}\left|F_{h}\right| \exp \left(i \varphi_{h}^{\mathrm{i}}\right) \exp (-2 \pi i h \cdot r) \\
& =2 \sum^{N / 2}\left|F_{h}\right| \cos \left(-2 \pi h \cdot r+\varphi_{h}^{\mathrm{i}}\right) \\
& =2 \sum^{N / 2}\left|F_{h}\right| \cos \left(-2 \pi h \cdot r+\pi+\varphi_{h}^{\mathrm{o}}\right) \\
& =-2 \sum^{N / 2}\left|F_{h}\right| \cos \left(-2 \pi h \cdot r+\varphi_{h}^{\mathrm{o}}\right) \\
& =-\rho^{\mathrm{o}}(r) .
\end{aligned}
$$

It will therefore represent the correct structure, but in negative contours. Again, the solvent flattening will fail to show any interpretable structural features.

If, in addition, the wrong handedness of the anomalous substructure is chosen, the SAD phase $\varphi_{\text {SAD }}^{\text {ic }}$ will have a negative value, as shown in Fig. $2(d)$. Taking into account that $\cos (-\varphi)=\cos (\varphi)$, the resulting map will have the following property:

$$
\begin{aligned}
\rho^{\mathrm{ic}}(r) & =\sum^{N}\left|F_{h}\right| \exp \left(i \varphi_{h}^{\mathrm{ic}}\right) \exp (-2 \pi i h \cdot r) \\
& =2 \sum^{N / 2}\left|F_{h}\right| \cos \left(-2 \pi h \cdot r+\varphi_{h}^{\mathrm{ic}}\right) \\
& =2 \sum^{N / 2}\left|F_{h}\right| \cos \left[2 \pi h \cdot(-r)-\varphi_{h}^{\mathrm{o}}\right] \\
& =2 \sum^{N / 2}\left|F_{h}\right| \cos \left[-2 \pi h \cdot(-r)+\varphi_{h}^{\mathrm{o}}\right] \\
& =\rho^{\mathrm{o}}(-r) .
\end{aligned}
$$

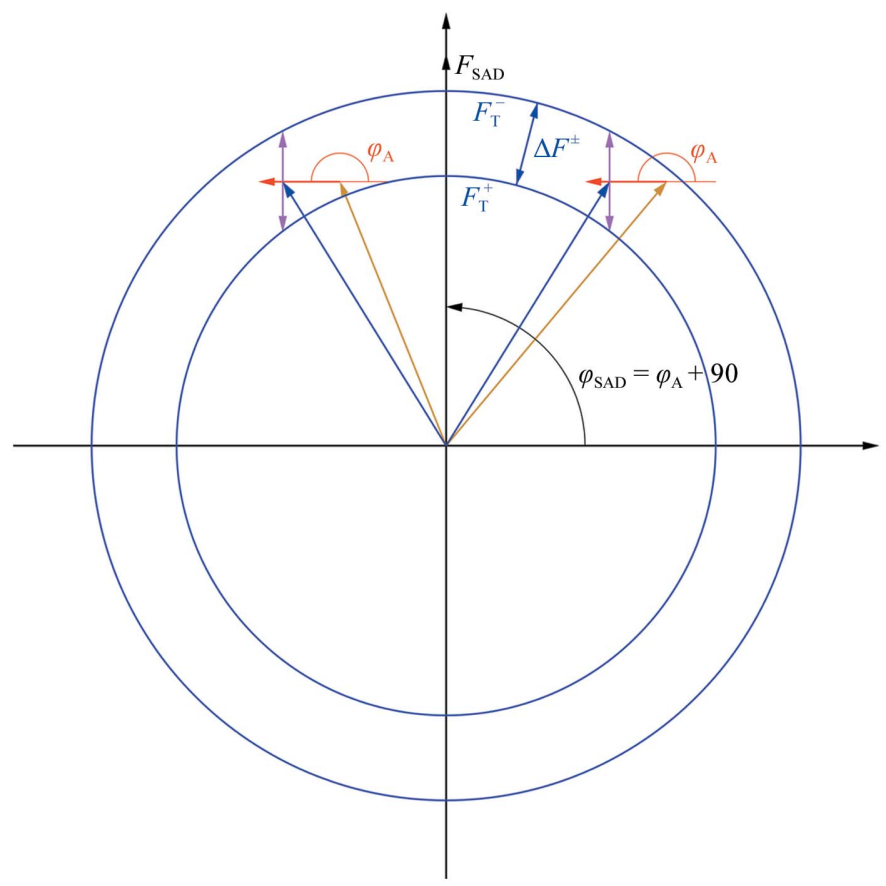

$(e)$

Figure 2 (continued)

(c) If only the signs of the anomalous differences are inverted, but the substructure has the correct handedness, the red/purple vectors are oriented in the same direction as originally, but the amplitudes of $F_{\mathrm{T}}^{+}$and $F_{\mathrm{T}}^{-}$(the radii of the blue circles) are exchanged, resulting in the SAD phase $\varphi_{\mathrm{SAD}}^{\mathrm{i}}=\pi+\varphi_{\mathrm{SAD}}^{\mathrm{o}}$ (d) If both the handedness of the substructure and the signs of the anomalous differences are wrong, the average SAD phase is negative with respect to the correct value, $\varphi_{\mathrm{SAD}}^{\mathrm{ic}}=-\varphi_{\mathrm{SAD}}^{\mathrm{o}}$. $(e)$ If the substructure is centrosymmetric, its normal structure factor does not have an imaginary component and the red vectors are horizontal. Two alternative SAD solutions lie symmetrically on both sides of the imaginary axis of the Argand diagram and the average vector is vertical with $\varphi_{\mathrm{SAD}}^{\mathrm{o}}= \pm \pi / 2$. Inversion of the substructure by the center has no effect (since it is already centrosymmetric), but exchange of the signs of anomalous differences changes the average phase to the negative value, $\varphi_{\mathrm{SAD}}^{\mathrm{i}}=-\varphi_{\mathrm{SAD}}^{\mathrm{o}}$, therefore inverting the handedness of all map features. 
This map will show the positive features, but inverted by the center of symmetry. The solvent-flattening procedure will enhance these features, but all chiral centers will have wrong handedness, helices in proteins will be left-handed etc.

\subsection{Centrosymmetric constellation of anomalous sites}

A special case may occur if all anomalous scatterers are centrosymmetically arranged in the crystal unit cell. One or two anomalous scatterer sites in the $P 1$ cell or one unique site in the $P 2_{1}$ cell are examples of this case. Another well known example is provided by the rhombohedral $R 3$ insulin crystal with two $\mathrm{Zn}$ atoms, both of which lie at the threefold axis (Dauter et al., 2002). The relevant phase diagram is shown in Fig. 2(e). As a consequence of the centrosymmetric arrangement of the anomalous sites, the red vector $F_{\mathrm{A}}$ must be horizontal, with its phase $\varphi_{\mathrm{A}}$ equal either to 0 or $\pi$ (if the center lies at the cell origin). The average phase $\varphi_{\mathrm{SAD}}$, which is always orthogonal to $\varphi_{\mathrm{A}}$, must therefore be equal to $\pm \pi / 2$. Since the substructure in this case is centrosymmetric, its inversion does not change anything; indeed, if $\varphi_{\mathrm{SAD}}= \pm \pi / 2$ then $\pi-\varphi_{\mathrm{SAD}}=$ $\pm \pi / 2$ as well and both conditions described above in $\S 2.1$ and $\S 2.2$ are fulfilled; therefore, $\rho^{\circ}(r)=-\rho^{\circ}(-r)$. If the anomalous signal is measured correctly, the Fourier map will be anticentrosymmetric and contain both the correct positive structural features and the inverted features in its negative contours. Such a map is therefore amenable to solvent flattening in the usual way. However, if the signs of the anomalous differences are exchanged [both cases in $\$ 2.3$ fulfilled, with $\rho^{\circ}(-r)=-\rho^{\circ}(r)$ ], the Fourier map will also be anti-centrosymmetric and contain correct features in the negative contours, but the positive features will have inverted chirality.

\section{MAD phasing}

Conceptually, multiwavelength anomalous diffraction phasing can be analyzed as the result of several SAD cases with different amounts of anomalous signal and this is illustrated in Fig. 3. Each wavelength indicates a pair of possible phase solutions (as in the SAD case), but only one solution in each pair coincides for all wavelengths. If more than one data set is available, there is no need to use the average 'SAD' phase; it is immediately possible to select proper values of the initial phases. In principle, the MAD map should show the correct structure with a low level of noise even before it is densitymodified.

The behavior of all individual phase estimations for all wavelengths after inversion of the anomalous signal or of the handedness of a substructure is therefore exactly the same as discussed in $\S 2$ for SAD phasing, with the same consequences for the map features.

\section{Isomorphous replacement phasing}

The isomorphous replacement method utilizes differences in the amplitudes measured from a native crystal and from one or more derivatives containing heavy atoms attached to the crystallized macromolecule. If only one derivative is used the technique is called single isomorphous replacement (SIR); in the case of more than one derivative it is called multiple isomorphous replacement (MIR). These phasing approaches do not make use of anomalous diffraction effects, even if the heavy atoms can potentially display anomalous scattering properties.

\subsection{SIR phasing}

The principle of SIR phasing is illustrated in Fig. 4(a). From the diffraction experiment, only the amplitudes of the native data and of one derivative data set are available. For a particular reflection it is initially only known that the end of the brown vector (representing the native protein amplitude $\left.F_{\mathrm{P}}\right)$ must lie on the Argand diagram at the circle of radius $\left|F_{\mathrm{P}}\right|$ and, analogously, that the blue vector corresponding to the derivative amplitude $F_{\mathrm{PH}}$ must end at the circle of radius $\left|F_{\mathrm{PH}}\right|$. The location of the heavy atoms can be obtained from Patterson or direct-methods calculations based on the observed isomorphous differences $\left(\left|F_{\mathrm{PH}}\right|-\left|F_{\mathrm{P}}\right|\right)$, since they are related to the heavy-atom amplitudes $F_{\mathrm{H}}$ through a trigonometric relation depending on the mutual disposition of $F_{\mathrm{P}}$ and $F_{\mathrm{H}}$, i.e. on the difference of the phases $\left(\varphi_{\mathrm{H}}-\varphi_{\mathrm{P}}\right)$. This is analogous to the relationship between $\Delta F^{ \pm}$and $F_{\mathrm{A}}$ in the $\mathrm{SAD}$ case. If the heavy-atom positions are known, it is possible to calculate their contribution $F_{\mathrm{H}}$ and locate on the Argand diagram two possible solutions of the protein phase.

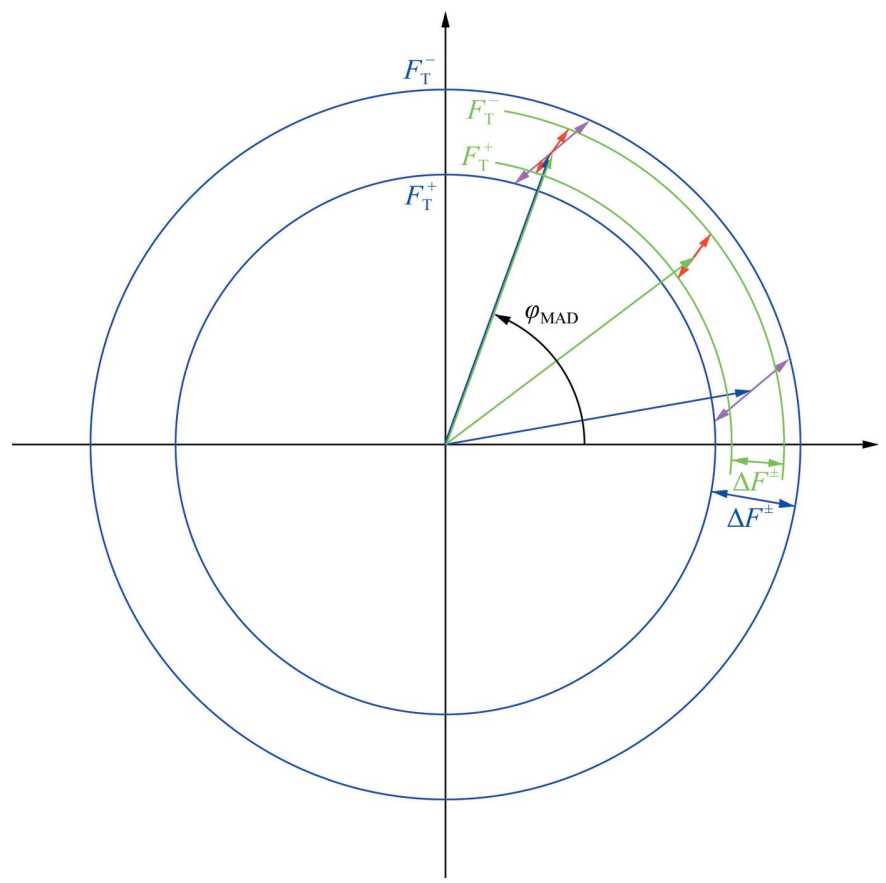

Figure 3

Two-wavelength MAD phases. Vectors corresponding to one wavelength are drawn in blue and purple and those corresponding to the second wavelength in green and red. Each wavelength indicates a pair of possible phases, as in the SAD case, but only one phase alternative for each wavelength coincides, indicating the correct solution for the protein phase. 
Since it is a priori not known which of them is correct, the 'average' phase $\varphi_{\text {SIR }}$ can be used for map calculation, which should show the correct image of the structure blurred by a degree of noise. A density-modification procedure may lead to an interpretable electron-density map and therefore to solution of the structure. This procedure is closely analogous to SAD phasing, except that in SIR the two alternative phase solutions are fully symmetric with respect to the lengths of all contributing vectors and the Sim probability contribution is

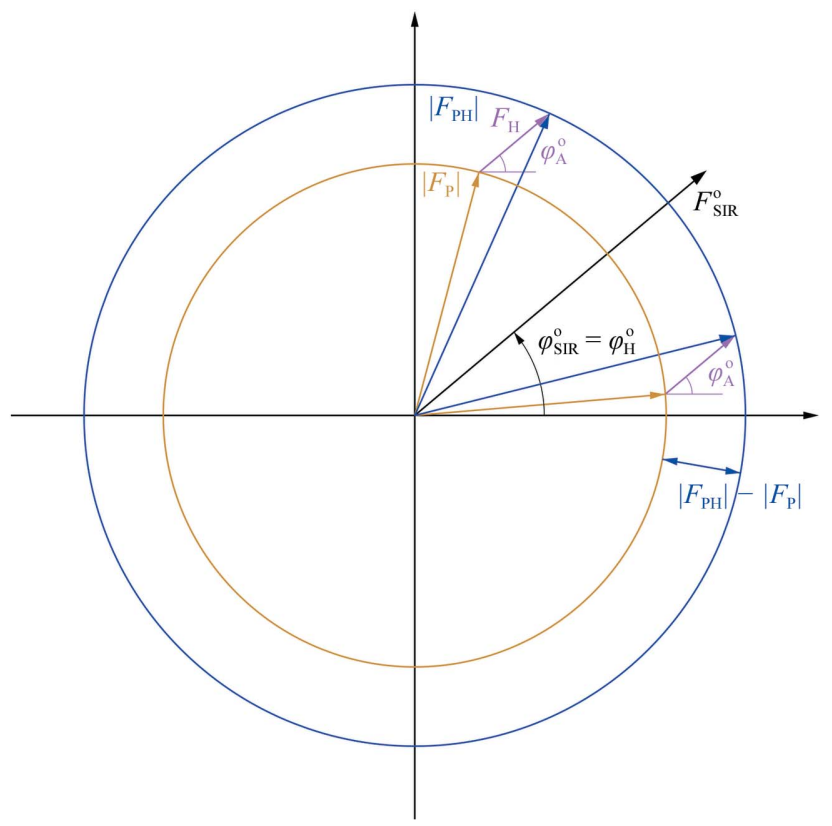

(a)

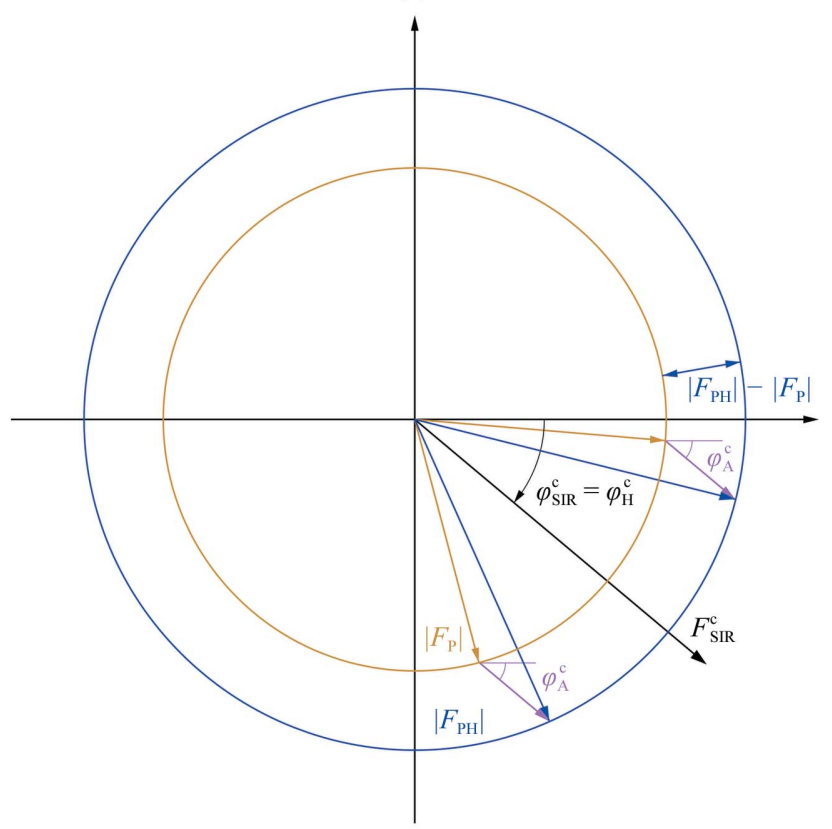

(b) equal for both possibilities. This results from the fact that in SIR the average phase $\varphi_{\text {SIR }}$ is the same as the substructure phase $\varphi_{\mathrm{A}}$, whereas in $\operatorname{SAD} \varphi_{\mathrm{SAD}}$ is shifted by $\pi / 2$ from $\varphi_{\mathrm{A}}$.

Since in the SIR approach the anomalous scattering effect is not used at all, it does not depend on the proper assignment of the signs of Bijvoet differences. However, the location of heavy atoms by Patterson or direct methods may equally probably lead to the correct or centrosymmetrically inverted constellation. If the substructure of heavy atoms has inverted handedness, the phase of its contribution, $\varphi_{\mathrm{H}}$, is negated, which is shown in Fig. 4(b). The resulting phases will also have opposite signs and the corresponding Fourier map will show the image of the structure inverted by the center of symmetry.

If the heavy atoms are arranged in the unit cell centrosymmetrically, the resulting SIR phases will have values of 0 or $\pi$. In this case the Fourier map will also be centrosymmetric and will contain two superimposed images of the structure. In this case density-modification procedures will not lead to the structure solution, in contrast to the situation with SAD/ MAD, where a centrosymmetric substructure results in the anti-centrosymmetric Fourier map.

\subsection{MIR phasing}

Similarly to the second-wavelength data in MAD, the second heavy-atom derivative data in MIR indicate which of the alternative SIR phases is correct, as illustrated in Fig. 4(c). If there are more derivatives, such an indication becomes stronger. It is assumed that the contributions of the various heavy atoms are not equivalent, since obviously for reliable phase indication the heavy-atom vectors (purple and red in

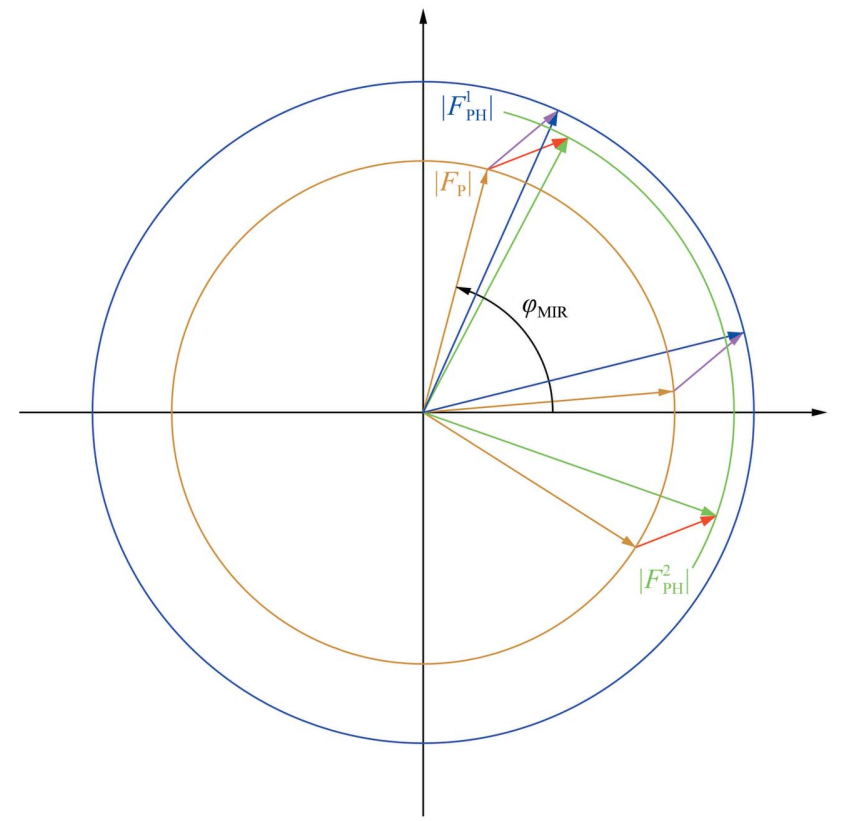

(c)

Figure 4

Phasing based on isomorphous signal. (a) SIR case, with the measured native protein amplitude shown in brown, the derivative amplitude in blue and the calculated contribution of the substructure in purple. There are two possible solutions and the initial average protein phase $\varphi_{\text {SIR }}^{\circ}$ corresponds to the phase of the heavy-atom substructure. (b) If the handedness of the substructure is inverted, the phase of the substructure as well as the average protein phase $\varphi_{\mathrm{SIR}}^{\mathrm{c}}$ are reversed, $\varphi_{\mathrm{SIR}}^{\mathrm{c}}=-\varphi_{\mathrm{SIR}}^{\mathrm{o}} .(c)$ If there is more than one derivative, it is possible to select the correct phase where the individual indications from all derivatives coincide. 
Fig. 4c) must differ. Moreover, the contribution of the macromolecule itself (represented by the brown vectors) must be the same in all the derivatives, which means that all the crystals should be isomorphous, hence the name of this method. If the introduction of heavy atoms affects the orientation of the protein molecule in the unit cell or causes changes in the crystal unit-cell parameters, the isomorphicity is no longer preserved and all MIR/SIR phase indications will be aberrated by errors. This effect is not so detrimental for the MAD/SAD techniques, where all data are usually collected from a single crystal. Obviously, all these phasing methods are negatively affected by inaccuracies in the measured diffraction intensities and by the radiation damage inflicted on crystals by exposure to X-rays.

The effect of wrong handedness of the heavy-atom substructure is the same as in the SIR case and results in positive but centrosymetrically inverted map features.

\section{Combination of isomorphous replacement and anomalous diffraction signals}

Fig. 5 illustrates the simplest case of SIRAS, with one derivative displaying a significant anomalous scattering effect. Individually, each SAD and SIR contribution leads to two possible phase solutions, but only one of them is common to both approaches, so that the phase indication in this case is in principle unequivocal. In general, in the MIRAS approach the consequences of incorrect assignment of anomalous differences or substructure may be more complicated than explained above. A simple summary is presented in Table 1.

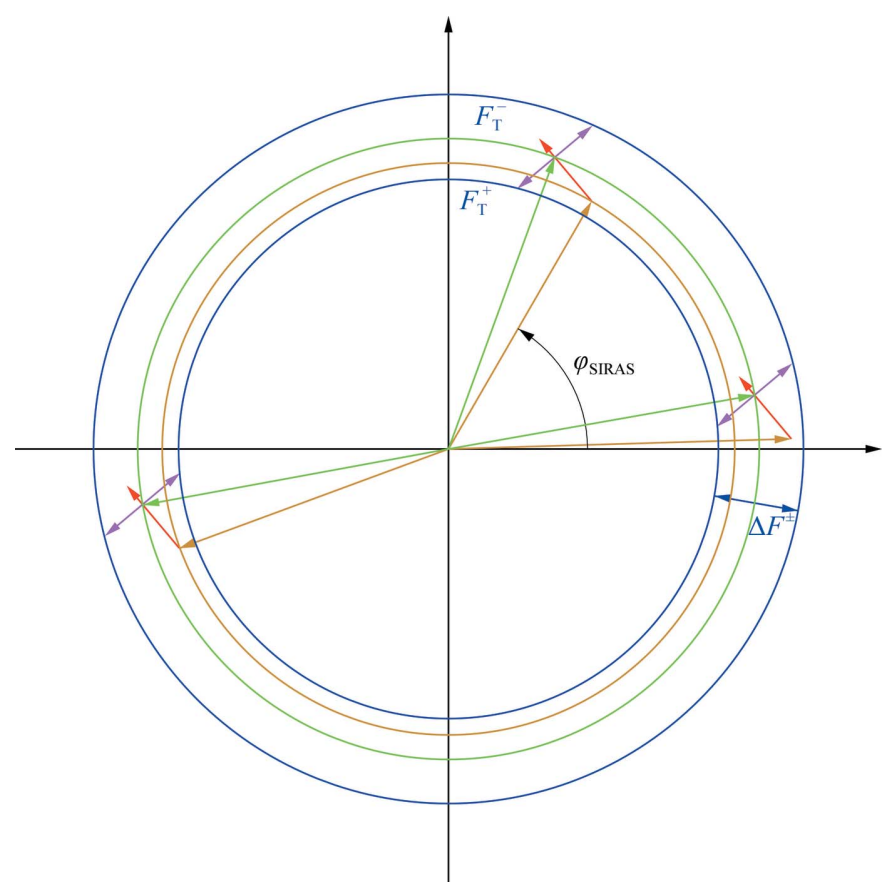

Figure 5

In the SIRAS approach, the isomorphous contribution provides a pair of phase indications and the anomalous contribution provides another pair of phase indications, but only one indication in each pair coincides, giving the correct protein phase.
Table 1

Phase estimations based on the anomalous or isomorphous signal for various combinations of substructure handedness and signs of anomalous differences.

\begin{tabular}{lllll}
\hline Handedness & Correct & Correct & Inverted & Inverted \\
\hline Sign of $\Delta F^{ \pm}$ & Positive & Negative & Positive & Negative \\
\hline SAD/MAD & $\varphi$ & $\pi+\varphi$ & $\pi-\varphi$ & $-\varphi$ \\
SIR/MIR & $\varphi$ & $\varphi$ & $-\varphi$ & $-\varphi$ \\
MIRAS & $\varphi$ & $?$ & $?$ & $-\varphi$ \\
\hline
\end{tabular}

Obviously, with correct signs of Bijvoet differences and proper handedness of the substructure, the Fourier map will represent the true crystal structure. However, another clear case results if the anomalous signs as well as the substructure are inverted, when the phase indications contributed by both isomorphous and anomalous differences are wrong but in agreement. The resulting Fourier map will show the image of the structure inverted by the center of symmetry.

In the two remaining combinations, the contributions of isomorphous and anomalous effects contradict each other. The resulting phases will depend on the relative strength of the two effects. If one of the sources of phasing significantly prevails, the Fourier map may resemble to some extent the correct structural features in its positive or negative contours. However, it is certainly best to make sure that the interpretation of the anomalous differences and of the substructure is correct.

\section{Conclusions}

Inversion of handedness of the anomalous substructure and change of sign of the anomalous differences result in a change of the phases of all reflections. The corresponding features of the Fourier maps calculated with such phases depend on the property of the trigonometric cosine function appearing in the Fourier synthesis in the following way:

\section{In SAD/MAD:}

Inverted handedness of substructure:

Exchanged signs of anomalous differences:

Inverted handedness and signs:

In SIR/MIR:

Inverted handedness of substructure:

Exchanged signs of anomalous differences:

$$
\begin{aligned}
& \rho^{\mathrm{c}}(r)=-\rho^{\mathrm{o}}(-r) \\
& \rho^{\mathrm{i}}(r)=-\rho^{\mathrm{o}}(r) \\
& \rho^{\mathrm{ic}}(r)=\rho^{\mathrm{o}}(-r)
\end{aligned}
$$$$
\rho^{c}(r)=\rho^{o}(-r)
$$

no effect
As clearly stated by Matthews (2007), in each of these cases it should be easily possible to identify the type of error at the early stage of map interpretation.

In the special case of a centrosymmetric constellation of the anomalous substructure, the problem of its handedness obviously does not exist and the phasing procedures should lead to the correct crystal structure. However, if the signs of the anomalous differences are changed, the map will show features with wrong handedness. 
The latter situation caused the unfortunate need to retract the wrongly interpreted structures of $\mathrm{ABC}$ and EmrE transporters (Chang et al., 2006). Two Os atoms in the $P 1$ cell form a centrosymmetric constellation, assuming comparable occupancies. The inadvertent exchange of the signs of all anomalous differences at the data-processing stage must have led to a nonrandom map that, however, exhibited inverted chirality. Thus, the only explanation of the origin of the incorrect model would be that the right-handed helices were actually built into the left-handed map features, something that would be possible in a map at low resolution.

All detectors and data-processing programs in current routine use produce data that are indexed in the correct righthanded coordinate system. Inversion of the anomalous signal may occur only as a result of using programs or procedures that have not been properly validated.

This work was supported in part by the Intramural Research Program of the NIH, National Cancer Institute, Center for Cancer Research and with Federal funds from the National Cancer Institute, National Institutes of Health under
Contract No. NO1-CO-12400. The content of this publication does not necessarily reflect the views or policies of the Department of Health and Human Services, nor does the mention of trade names, commercial products or organizations imply endorsement by the US Government.

\section{References}

Blundell, T. L. \& Johnson, L. N. (1976). Protein Crystallography. New York: Academic Press.

Chang, G., Roth, C. B., Reyes, C. L., Pornillos, O., Chen, Y.-J. \& Chen, A. P. (2006). Science, 314, 1875.

Dauter, Z. (2002). Acta Cryst. D58, 1958-1967.

Dauter, Z., Dauter, M. \& Dodson, E. J. (2002). Acta Cryst. D58, 494-506.

Drenth, J. (1999). Principles of Protein X-ray Crystallography, 2nd ed. Heidelberg: Springer.

Matthews, B. W. (2007). Protein Sci. 16, 1013-1016.

Miller, G. (2007). Science, 314, 1856-1857.

Ramachandran, G. N. \& Raman, S. (1956). Curr. Sci. 25, 348-351.

Sim, G. A. (1959). Acta Cryst. 12, 813-815.

Sim, G. A. (1964). Acta Cryst. 17, 1072-1073.

Wang, B.-C. (1985). Methods Enzymol. 115, 90-112. 